\title{
10-nm resolution by magnetic force microscopy on FeNdB
}

\author{
P. Grüter, a) Th. Jung, H. Heinzelmam, A. Wadas, E. Meyer, H.-R. Hidber, \\ and H.-J. Guntherodt \\ Institute of Physics, Klingelbergstrasse 82, CH-4056 Basel, Switzerland
}

(Received 9 August 1989; accepted for publication 13 October 1989)

\begin{abstract}
Magnetic force microscopy (MFM) images of rapidly quenched FeNdB are presented. The magnetic domain structure as observed by MFM consists of elongated, polyhedral-shaped domains imaged by measuring forces smaller than $10^{-9} \mathrm{~N}$ at distances ranging from 20 to more than $200 \mathrm{~nm}$. The domain transition regions, which are quite sharp and well defined, often show a double-peaked structure with a peak-to-peak distance of $10 \mathrm{~mm}$. At force sensor tip-to-sample separations larger than $90 \mathrm{~nm}$ only a single peak is observable. It has to be assumed that the relevant effective magnetic volume of the force sensing tip is considerably smaller than the geometric dimensions as determined by scanning electron microscopy in order to inderstand this $10-\mathrm{nm}$ resolution.
\end{abstract}

\section{INTRODUCTION}

Much attention has recently been paid to FeNdB alloys that exhibit a higher-energy product than that of other commercially available permanent magnets such as CoSm. Permanent magnets based on the FeNdB system are produced by two distinctly different methods: rapid solidification processing (mostly melt-spinning) followed by hot pressing and hot deformation, or powder metallurgy combined with thermal treatments (sintering).

The ribbon samples produced by melt spinning are considered as single-phase materials as compared to sintered magnets which are considered multiphase. One of the most debated questions is whether a domain nucleation or a domain-wall-pinning mechanism is responsible for the high coercivities (in excess of $10 \mathrm{kOe}$ ) and high-energy products (more than $13 \mathrm{MGOe}$ ) in these materials. A recent review ${ }^{1}$ examines the validity of the nucleation and domain-wallpinning models. It is concluded that the model of strong pinning of domain walls both in rapid solidified and sintered magnets accounts for the observed magnetic properties. Direct verification of this model and characterization of the domain-wall-pinning sites with high spatial resolution would clearly be important.

Lorentz microscopy ${ }^{1-4}$ has been used to image domains in melt-spun FeNdB in order to correlate microstructure and magnetic properties. Domains have a polyhedron shape and are slightly elongated with an average length of more than a micron and widths between 200 and $700 \mathrm{~nm}$.

Recently, force microscopy ${ }^{5}$ has been applied to the study of magnetic samples on a submicron scale. ${ }^{6,7}$ The force sensor in magnetic force microscopy (MFM) consists of a sharp ferromagnetic tip mounted on a cantilever. Forces acting on the tip cause a deflection of the cantilever, which can be measured by electron tunneling, ${ }^{5.7}$ or result in a change of resonance frequency, which can be detected by interferometry, ${ }^{6,8}$ piezoelectrically ${ }^{9}$ or capacitively. ${ }^{10}$ The measured interaction forces depend on the magnetic struc-

\footnotetext{
") Present address: IBM Research Division, Almaden Research Center, 650 Harry Road, San Jose, CA 95120-6099.
}

ture of the sample and the physical properties-such as magnetization and geometry-of the tip. ${ }^{16,12}$ Measurable magnetic forces are a result of gradients of magnetization ${ }^{7}$ or are associated with local changes of the sample (or tip) magnetic moments induced by the magnetic ficld of the tip or sample, respectively. ${ }^{13}$ Several questions motivated the MFM study on this material:

(1) Can the domain strueture of $\mathrm{FeNdB}$ be observed by MFM? The possibly strong stray fields of the sample might infuence the force-sensor magnetization irreversibly, thus not allowing reproducible imaging.

(2) What is the ultimate experimental lateral resolution of MFM? The domain walls in FeNdB are known to be very narrow-only $3 \mathrm{~nm}^{2}$

(3) The extreme magnetic properties (large crystal anisotropy, large saturation magnetization $M_{s}$, large coercitive field $H_{c}$, very small domain-wall widths) might lead to previously unobserved phenomena, the explanation of which would motivate one to think more about the technique of MFM with subsequent improvements in theory and experiment.

\section{METHODS AND CHARACTERIZATION}

The instrument used in our experiments is described in detail in a previous paper. ${ }^{14}$ th was operated both in the equiforce mode and in the variable deflection mode when measuring magnetic structures, and in the regime of repulsive contact forces in the variable deffection mode when topographic information was needed. Images were typically obtained in $60 \mathrm{~s}$ by scanning the sample in $x$ and $y$ directions, and simultaneously measuring the cantilever deffection in $z$. All measurements were performed in ambient air on a passively dampened optical table.

Magnetic force sensors were cut from $10-\mu \mathrm{m} \mathrm{Ni}$ foil and electrochemically etched to a tip of less than 100-nm radius with an apex angle of $10^{\circ}$ at one end. They were not subjected to any magnetic pretreatment. Typical force constants of the lever are $0.1 \mathrm{~N} / \mathrm{m}$. Some topographic measurements were done with microfabricated $\mathrm{SiO}_{2}$ force sensors. Instrumental noise corresponds to less than $0.1-\mathrm{nm}$ cantilever deflection.

Calculations of the lines of force were done by the same 
method as presented in previous publications (see Ref. 12 and references therein). Analytical formulas describe the magnetic field above a sample of constant thickness with a periodic domain structure; transitions between domains are modeled as being infinitely sharp. The tip-sample interaction can then be calculated by assuming a truncated pyramid as a tip model. ${ }^{12}$ A domain structure can be incorporated in our tip model.

The alloy composition of $\mathrm{Fe}_{81.3} \mathrm{Nd}_{13.5} \mathrm{~B}_{5.2}$ was quenched at a wheel speed of $21 \mathrm{~m} / \mathrm{s}$ to obtain ribbons having optimum magnetic properties (energy product 11.5 MGOe). No further heat treatments were made. This sample was previously measured by STM in UHV, ${ }^{15}$ clearly showing faceted spheroidal grains $20 \mathrm{~nm}$ in size after $A r$ ion etching. The sample was characterized in air by $A F M$ as the oxidized surface layer is insulating. No sign of small grains could be observed; only fairly flat large scale variations are detectable on a submicron scale (Fig. 1).

\section{H. RESULTS}

Polyhedral-shaped structures with characteristic lengths of $600-700 \mathrm{~nm}$ are observed by MFM on this sample. Very similar images are obtained for changes in the interaction distance of up to $225 \mathrm{~nm}$; larger separations were not attempted due to experimental constraints, i.e., the danger of depolarizing the seanning piezo elements (Fig. 2). The structures become sharper and more pronounced the smaller the force sensor-sample separation is. This can be observed best by comparing single scan lines (Fig. 2).

The magnetic nature of the image-generating forces is proven by measuring the attractive interaction force as a function of force sensor-sample separation. Attractive forces in the range of $n \mathbb{N}$ can be measured up to separations of more than $200 \mathrm{~nm}$, thus precluding van der Waals or electrostatic forces, which are generally shorter ranged. Higherresolution images obtained at comparatively close distances $z$ of about $35 \mathrm{~nm}$ show that the polyhedral-shaped structures are separated by quite sharp, rather straight, and narrow transitions (Fig. 3). Furthermore, some smoother, hill-like structures with typically $80 \mathrm{~nm}$ diameter are observable. Single line scans show these two types of high-resolution structures clearer.

These single scan lines of the sharp transitions also often show an interesting double-peak substructure with a peak-

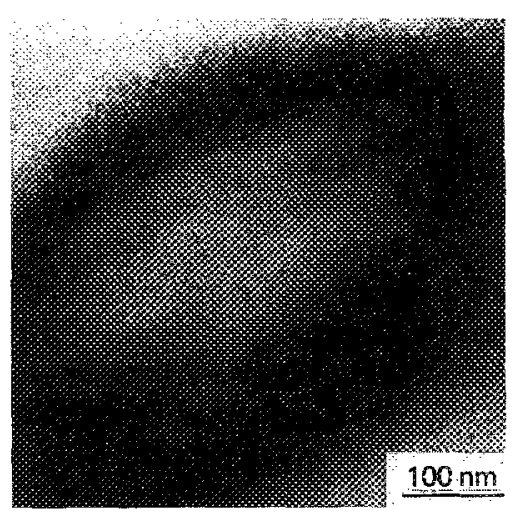

FIG. 1. AFM image of melt-spun FeNdB acquired in the variable deflection, repulsive contact mode of operation. The side length of the figure is $500 \mathrm{~nm}$, the total $z$ scale corresponds to $30 \mathrm{~mm}$.
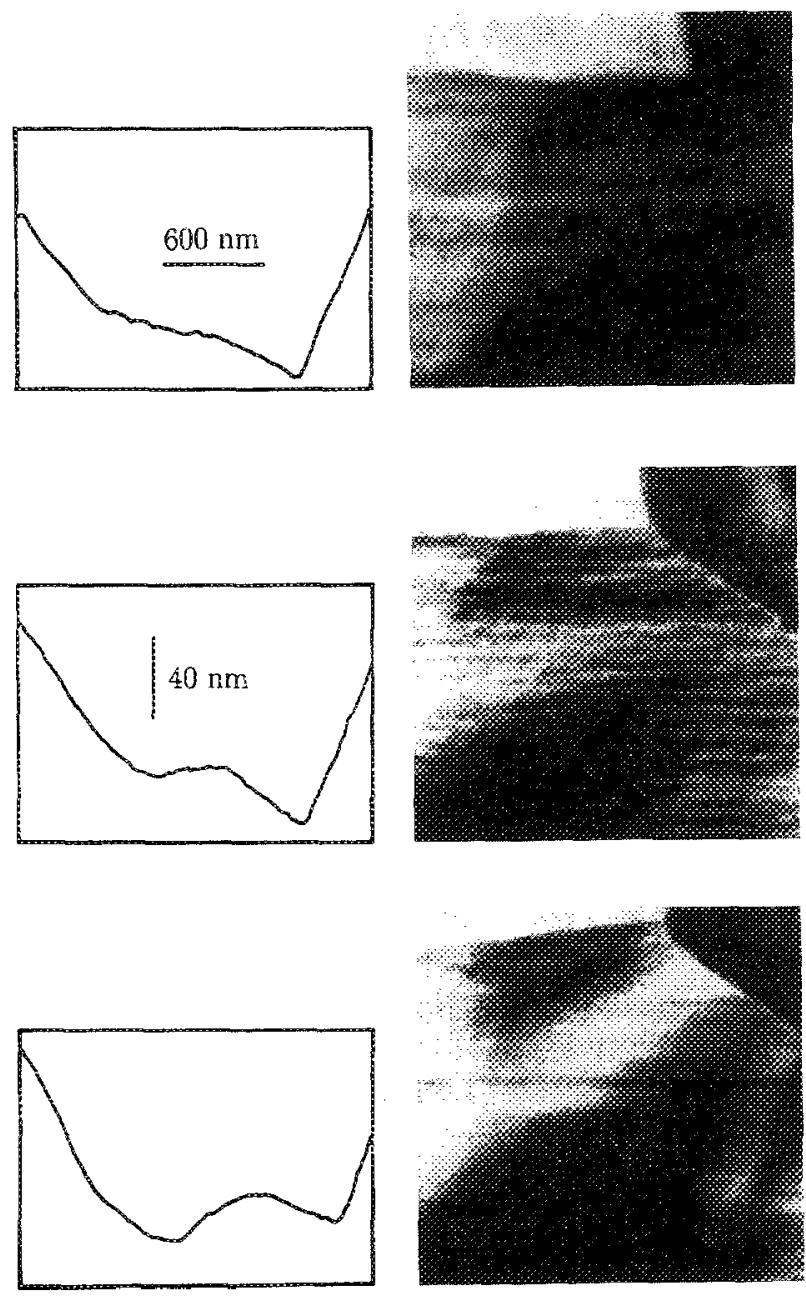

FIG. 2. Polyhedral-shaped domains measured by MFM on meit-spun FeNdB. The figure shows images with a side length of $2.1 \mu \mathrm{m}$. The second and third images were measured 125 and $250 \mathrm{~nm}$ closer to the sample than the top image. Single scan lines demonstrate how the observed structures become sharper and more pronounced the smaller the force-sensor-to-sample separation is.

to-peak distance of about $10 \mathrm{~nm}$. This substructure is not observable on scans of an image otherwise perfectly reproduced and measured with a sample force-senscr separation increased by $90 \mathrm{~nm}$; only a single peak is observable.

Previously, highest-resolution MFM measurements obtained with a different force sensor in the variable deflection mode also showed a very similar doublc-peak structure (Fig. 4). ${ }^{16}$ MFM images of this double-peak structure have a resolution of better than $10 \mathrm{~nm}$, the absolute number depending on the definition [peak-peak separation or full width at half maximum (FWHM) of a single peak]. Similar doublepeaked structures were observed with difierent magnetic force sensors but never in the topographic imaging mode.

\section{DISCUSSION}

The observable polyhedral structures with characteristic dimensions of about $600-700 \mathrm{~nm}$ are attributed to the magnetic domain structure of the sample as the forces acting are long ranged, predominantly magnetic in nature, and no evident similarity to topography is observable. Further- 


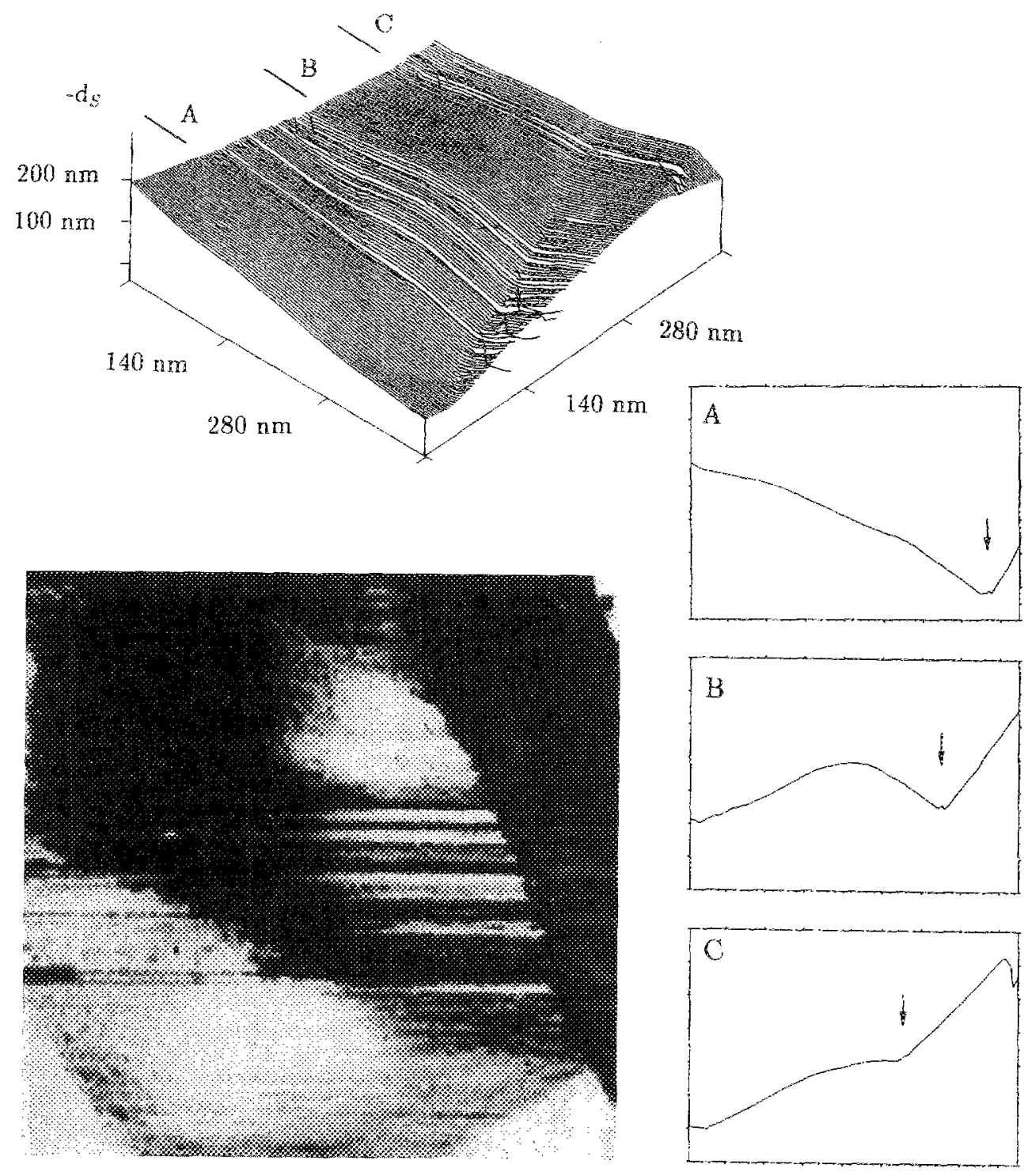

EIG. 3. MFM image of melt-spun FeNdB measured at a force-sensor-sample separation of about $35 \mathrm{~nm}$. The bottom image is a processed version of the line scan image. A dowble-peaked structure often observed at domain transition regions is marked by arrows on the single scan lines.

more, Lorentz microscopy images of the domain structure look very similar to these MFM images.

The reproducibility of images demonstrates that the Ni force sensor magnetic structure was not irreversibly changed during scanning. This shows that the induced coercivity of the tip due to shape anisotropy is large, as the FeNdB stray field is expected to be of considerable magnitude. Empirical$1 y$, it can be stated that the infuence of the sample stray field on the tip magnetic structure is too small to induce irreproducible domain changes.

For relatively hard ferromagnetic materials with $H_{k} / M_{s} \gg 1$, such as FeNdB, the structural surface topography does not significantly infuence the micromagnetic distribution. As a consequence, parts of the sample where the surface is not orientated parailel to the local spin distribution are magnetically charged. Thus, the surface roughness causes an overall stray-field background which might be detected by MFM and would explain the large-scale, 80 nm hills observed in MFM images. "t

The most surprising experimental observation on FeNdB are the double peak structures separated by less than $10 \mathrm{~nm}$. These structures are due to magnetic forces with a high probability and related to the strong and Localized stray fields present at domain boundaries, as they are observable as far as $90 \mathrm{~nm}$ from the sample surface, thus excluding the possibility of image contrast generated by van der Waals or electrostatic forces. Furthermore, no similar siructures have been observed in topographic images. A conclusive interpretation of these double peak structures cannot be given presently; three experimental facts would have to be explained: (1) the observed resolution of $10 \mathrm{~nm}$; (2) the double-peak structure with a force amplitude of about $0.6 \mathrm{nN}$ at an estimated distance of $20 \mathrm{~mm}$; (3) at separations larger than 90 $n m$ only a single-peak structure is observable.

Different possibilities might explain some of these facts, but we have not succeded yet to combine all and thus simblate the experimental results. The major challenge is to understand and simulate the $10-n m$ resolution when imaging domain transition regions in $\mathrm{FeNdB}$.

It is improbable that the double-peak structure within the domain transition region can be interpreted as arising from a pair of closely spaced parallel domain walis, as this is an energetically unfavorable configuration in the absence of a very strong external magnetic field. 


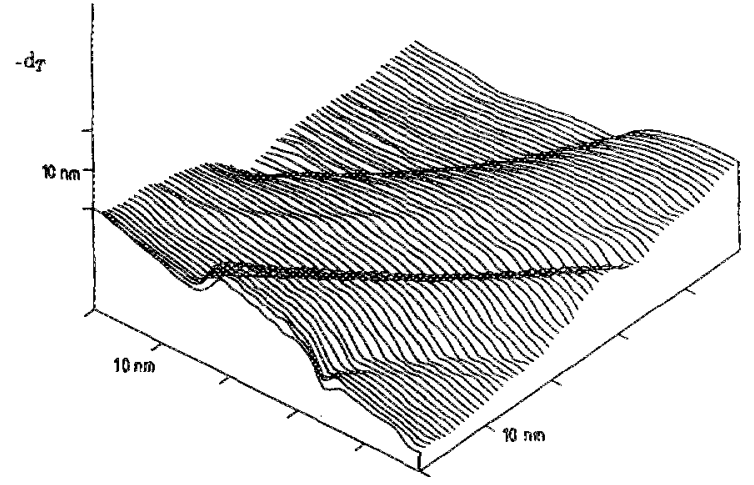

$-d_{f}[n m]$

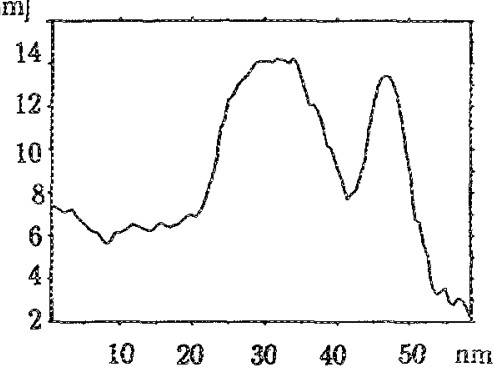

FlG. 4. Ultrahigh resolution MFM image of a domain transition region acquired in the variable deflection mode on FeNdB.

Simulations show that in order to obtain a 50-nm resolution with a homogeneously magnetized force-sensor tip the geometry has to be nearly cylindrical with a length of $300 \mathrm{~nm}$ and a radius of $20 \mathrm{~nm} .{ }^{12}$ A tip giving a higher resolution would have to have an even smaller radius and the same length, which seems quite unreasonable in view of characterization of force sersors by SEM, and would also be mechan:cally unstable. One can speculate that the balancing effects of exchange, magnetocrystalline, and magnetostatic energy contributions, which lead to a nonhomogeneous magnetization $M(r)$ of the tip especially at rough surfaces, would result in an effective nonhomogeneous magnetization $M(\mathbf{r})$ of the tip with a smaller spatial extent than the geometric tip volume [Fig. 5(a)]. The spatial extent of the effective magnetization, leading to the measurable force contrast, might be much smaller than the geometric dimension of the tip as the volume of the relevant effective magnetization is a function of the characteristic decay length of the magnetic sample under investigation. This decay length is of the order of tens of nanometers for domain walls in $F \mathrm{eNdB}$ and might explain the disappearance of the double-peaked structure for separations larger than $90 \mathrm{~nm}$. This concept of an effective magnetic volume can best be viewed by introducing magnetic charges, defined as $\rho_{\mathrm{mag}}=\nabla \cdot \mathrm{M}$ or $\sigma_{\mathrm{mag}}=\mathrm{n} \cdot \mathrm{M}$; a nonhomogenous magnetization distribution thus leads to a nonhomogeneous charge distribution [Fig. 5(a)]. Forces are the result of the interaction of these magnetic charges with the sample magnetic stray field. Furthermore, as has been emphasized by Hartmann, ${ }^{17}$ the widnh of a certain structure in an MFM image does not always increase with increasing tip volume. A detailed rigorous calculation of $\mathrm{M}(\mathrm{r})$ for an arbitrary tip geometry has not been successfully performed yet. $^{18}$ (a)
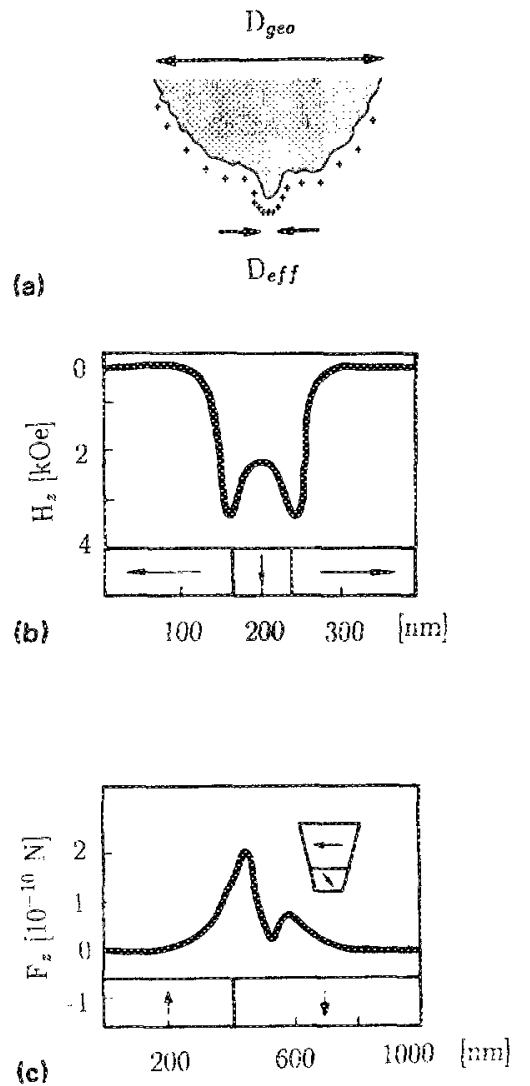

(c)
FiG. 5. (a) Model of a tionhomogeneously magnetized tip with a diameter $D_{\text {sll }}$ much smaller than the geometric diameter $D_{\mathrm{gec}}$. Magnetic charges are indicated, strongly localized at an asperity. The magnetic image contrast will be dominated by the effective magnetic volume with the characteristic dimension $D_{\text {ur }}$ in the tip is scanned close enough to the sample and the decay length of the magnetic structure under investigation is comparable to $D_{\text {erf }}$. (b) Calculated $z$ component of $H$, for the magnetic sample structure indicated at a separation of $5 \mathrm{~nm}$ shows two peaks. (c) The characteristics of double-peak structures depends on the model tip domains used in the simulation. The tip used here, modeled as a truncated pyramid (inset), has a lower diameter of 60 $\mathrm{nm}$, a length of $300 \mathrm{~nm}$, and a top diameter of $180 \mathrm{~nm}$. The two tip domains are 70 and $230 \mathrm{~nm}$ long. Details of parameters can be found in Ref. 20.

The following examples serve as an illustration of two distinctly different mechanisms leading to double-peaked structures. In simulations the input parameters are usually determined from electron microscopy images of the tip; these numbers are considerably larger than those necessary to achieve a $10-\mathrm{nm}$ resolution in simulations. As the issue of an "effective" tip magnetization volume is far from settled we will not attempt a quantitative simulation. This could be done by taking the effective tip magnetization volume as a fit parameter. In the following simulations an effective tip diameter of $80 \mathrm{~nm}$ is assumed.

Double-peak structures can be simulated either with a simple sample magnetic structure not unrealistic for FeNdB [Fig. 5(b)] or a simple, nonhomogeneous magnetization $M(r)$ of the force-sensor tip indicated in the inset [Fig. $5(\mathrm{c})$ ]. It has to be pointed out, however, that in both simulations the resolution is limited by the tip geometry and is thus at least about an order of magnitude larger than experimentally observed. These two mechanisms can explain the observed double-peak structure if an effective spatial extent of the magnetization substantially smaller than the geometric dimension of the tip is assumed. Clearly there is a need to experimentally measure and theoretically determine the magnetization of sharp tips employed in MFM in the future.

The distance dependence of the observed double-peak structure might be attributed to the characteristic decay length of the sample magnetic structure and the spatial extent of the effective tip magnetic structure.

It is clear that presently the sample and force-sensor tip effects cannot be deconvoluted and thus a conclusive interpretation of the double-peak structure cannot be given. The 
width of $10 \mathrm{~nm}$ could be determined either by the sample specific magnetic structure, the force-sensor tip, or (most probable) a convolution of both. Thus, at this stage it cannot be decided what the ultimate sample specific lateral resolution of MFM is. ${ }^{19,20}$ Irrespective of this issue, it can be stated that it is possible to obtain magnetic information with $10 \mathrm{-nm}$ lateral resolution by the technique of MFM. It can be concluded that the interpretation of $\mathrm{MFM}$ images on a scale smaller than $100 \mathrm{~nm}$ is presently not straightforward; exter:sive image simulation with detailed knowledge of the tip magnetization $M(r)$ is necessary.

\section{SUNMARY}

In summary, we can conclude the following:

(1) The magnetic domain structure of rapidly quenched FeNdB can be imaged by MFM in air.

(2) In principle a very high lateral magnetic resolution of $10 \mathrm{~nm}$ is achievable by the technique of MFM.

(3) Detailed interpretation of such high-resolution measurements is currently limited due to the unknown tip magnetic structure. Future developments in the theoretical understanding and the fabrication of magnetic force-sensor tips are necessary.

\section{ACKNONEEDGMENTS}

We thank T. R. Albrecht from Stanford University for his assistance with the $\mathrm{SiO}_{2}$ force-sensor microfabrication and R. Guggenheim and M. Düggelin from the University of Basel for the force-sensor characterization by SEM. This research is supported by the Swiss National Science Founda- tion and the Komission zur Forderung der Wissenschaftlichen Forschung.

${ }^{1}$ G. C. Hadjpanayis and A. Kim, J. Appl. Phys. 63, 3310 (1988).

${ }^{2}$ R. K. Mishra, J. Magn. Magn. Mater. 54-57, 450 (1986).

${ }^{3}$ G. C. Hadjipanayis, Y. F. Tao, and K. R. Lawless in Procedings of the 4th International Symposium on Magnetic Anisotropy and Coercivity in Rare Earth-Transition Metal Alloys, edited by K. Sirnat, Dayton (1985), p. 657 .

4G. C. Hadjiparayis, R. C. Dickenson, and K. R. Lawless, J. Magn. Magn. Mater. 54-57, 557 (1986).

${ }^{5}$ G. Binnig, C. F. Quate, and Ch. Gerber, Whys. Rev. Lett. 55, 930 (1986)

b Y. Martin and K. K. Wickramasinghe, Appl. Phys. Lett. 50, 1455 (1987).

${ }^{7}$ J. J. Sáenz, N. Garcia, P. Grütter, E. Meyer, H. Heinzelmann, R. Wiesendanger, L. Rosenthaler, H.-R. Hidber, and H.-J. Güntherodt, J. Appl. Thys. 62, 4293 (1987).

D. Rugar, H. J. Mamin, R. Eriandsson, J. E. Stern, and B. D. Terris, Rev. Sci. Instrum. 59, 2337 (1988).

${ }^{9} T$. Göddenhenrich, U. Hartmann, M. Anders, and C. Heiden, B. Microse. 152,527 (1988).

10 T. Göddenhenrich, H. Lemke, U. Hartmann, and C. Heiden, J. Vac. Sci. Technol. A (to be published).

${ }^{11}$ U. Hartmant, J. Appi. Phys. 64, 156 (1988).

${ }^{12}$ A. Wadas and P. Gritter, Phys. Rev. B 39, 12013 (1989).

${ }^{13}$ J. J. Sáenz, N. Garcia, and J. C. Slonczewski, Appl. Plys. Lett. 53, 1449 (1988).

${ }^{14}$ H. Heirtelmann, P. Groitter, E. Veyer, L. Rosenthaler, H. R. Hiber, and H. J. Güntherodt, Surf. Sci. 183/199, 29 (1987).

${ }^{5}$ B. W. Corb, M. Ringger, H.- Gütherodt, and $\mathrm{W}$. E. Pinkerton, Appl. Phys, Lett. 30,333 (1987).

${ }^{16}$ P. Grütter, E. Meyer, H. Heinzelmann, L. Rosenthaler, H. R. Hidber, and H.-J. Güntherodt, J. Vac. Sci. Technoi. A 6,279 (1988),

${ }^{17}$ U. Hartmann, Phys. Lett. A 137, 475 (1989).

${ }^{18}$ A. Hubert (private cormmunication).

${ }^{19}$ P. Grütter, Ph.D. Thesis, University of Basel (1989).

${ }^{20}$ P. Grüter, A. Wadas, E. Meyer, H. Heinzelmann, H. R. Hidber, and H.J. Güntherodt, J. Vac. Sci. Technol. A (to be published). 